\title{
Economics and Practice
}

Die Gesundheitsökonomie, der sich das vorliegende Heft der Psychiatrischen Praxis widmet, gehört sowohl in der öffentlichen Diskussion als auch im Bereich der Forschung zu den gegenwärtig besonders heftig umstrittenen Themen. Dabei beginnen die Meinungsverschiedenheiten schon bei der Begriffsdefinition.

Als auf der wissenschaftlichen Jahrestagung des Vereins für Socialpolitik die Frage nach einer Definition für Gesundheitsökonomie gestellt wurde, war die Antwort: „1. Gesundheitsökonomie ist das, was Gesundheitsökonomen machen; und 2. ein Gesundheitsökonom ist keiner, der die Gesundheitsausgaben schon deshalb für zu hoch hält, weil sie zu hoch sind“ ([1], S. 657).

In Anbetracht des offensichtlichen Fehlens einer einheitlichen Begriffsdefinition kommt Graf von der Schulenburg, einer der renommiertesten Gesundheitsökonomen Deutschlands, zu dem Schluss: „Gesundheitsökonomie ist einfach die Analyse der wirtschaftlichen Aspekte des Gesundheitswesens unter Verwendung von Konzepten der ökonomischen Theorie“ ([2], S. 16). Dagegen betont Reiner Leidl: „Gesundheitsökonomie ist aber keine Kostendämpfungslehre - obwohl sie die Gründe des Kostenwachstums kennen und die Wirkung von Dämpfungsmaßnahmen aufzeigen können muss“ ([3], S. 78). Am weitesten vor wagt sich jedoch Deutschlands prominentester Gesundheitsökonom, Karl Lauterbach, wenn er feststellt: „Gesundheitsökonomische Analysen verbessern die Struktur, Prozess- und Ergebnisqualität der Versorgung“([4], S. 121).

Die aktuellen Entwicklungen im Gesundheitswesen lassen jedoch einige Zweifel daran aufkommen, ob die Gesundheitsökonomie in der Praxis tatsächlich zu einer Verbesserung der medizinischen Versorgung beitragen kann, oder ob sie nicht eher für
Kostendämpfungsmaßnahmen instrumentalisiert wird. Schließlich werden originäre gesundheitsökonomische Erkenntnisse, die auf eine effiziente Gesundheitsversorgung und eine optimale Ressourcenallokation zielen, im klinischen Alltag derzeit so gut wie nicht berücksichtigt [5]. Was momentan politisch und praktisch umgesetzt wird, sind Vorschläge zur Rationierung, nicht aber zur Rationalisierung.

Ein Beispiel ist die ambulante Soziotherapie, die nach den Erfahrungen eines Modellprojekts die Lebensqualität chronisch psychisch Kranker verbessert und gleichzeitig zu einer Kostenersparnis führt, die das drei- bis sechsfache der Soziotherapiekosten beträgt [6]. Trotzdem ist dieses Therapiekonzept bislang nur ein „Papiertiger“ geblieben [7]. Es kann zwar seit dem 1.1.2002 verordnet werden, aber die Zugangsvoraussetzungen der Kassen sind so hoch, dass bisher fast alle Anträge potenzieller Anbieter scheiterten. Es ist daher zu befürchten, dass eine flächendeckende Einführung der ambulanten Soziotherapie nicht gelingen wird.

Damit tut sich die Frage auf, warum sich die Umsetzung gesundheitsökonomischer Erkenntnisse in der Praxis so schwierig gestaltet. Blockieren die Kassen so offensichtlich effiziente Maßnahmen wie die ambulante Soziotherapie etwa deshalb, weil sie nicht daran glauben, in der Routineversorgung tatsächlich die prognostizierten Einsparungen erzielen zu können?

Für diese Vermutung würde zum einen sprechen, dass gesundheitsökonomische Untersuchungen in der Regel in einem speziellen Kontext durchgeführt werden, so dass die Übertragung der Ergebnisse auf andere Regionen, einen anderen Fallmix oder anders umgesetzte Interventionsstrategien ausgesprochen schwierig ist $[8,9]$. Erschwerend kommt in Deutschland hinzu, 
dass nur relativ ungenaue epidemiologische Daten verfügbar sind, und dass die Fragmentierung des Versorgungssystems die Ermittlung der tatsächlichen Behandlungskosten kompliziert macht [10].

Ein wichtiger Punkt ist zudem, dass gesundheitsökonomische Analysen noch zu selten berücksichtigen, dass die Wirksamkeit therapeutischer Maßnahmen unter experimentellen Bedingungen meist deutlich höher ist, als im klinischen Routineeinsatz [11 - 13]. Dabei spielt vor allem eine Rolle, dass die Compliance der Patienten unter experimentellen Bedingungen besser ist, und dass Therapieabbrecher in der Regel von der Auswertung ausgeschlossen werden. Überdies wird vermutet, dass auch das medizinische Personal in Modellprojekten engagierter ist als im klinischen Alltag [12].

Auch die große Heterogenität und die teilweise geringe methodische Qualität gesundheitsökonomischer Untersuchungen beeinträchtigen die Akzeptanz bei den Entscheidungsträgern im Gesundheitswesen [14]. So gibt es beispielsweise keine einheitliche Methode um eines der wichtigsten gesundheitsökonomischen Outcomekriterien - die Lebensqualität der Patienten - zu bestimmen. Daher verändert sich, je nachdem für welche Methode man sich entscheidet, auch das Ergebnis [15]. Deshalb wird immer wieder gefordert, bessere methodische Standards zu etablieren, die Vergleiche zwischen unterschiedlichen Analysen ermöglichen und die wissenschaftliche Qualität der Studien sichern [16]. Dabei handelt es sich jedoch um eine Gratwanderung, da eine stärkere Standardisierung auch negative Auswirkungen haben kann, indem sie neue Untersuchungsansätze in einem noch entwicklungsbedürftigen Fachgebiet unterdrückt.

Die bislang genannten Kritikpunkte, die eine Umsetzung gesundheitsökonomischer Erkenntnisse in die Praxis erschweren, liegen hauptsächlich in der Verantwortung der Wissenschaftler, die gesundheitsökonomische Untersuchungen durchführen. Mindestens ebenso hinderlich für den Praxistransfer sind jedoch strukturelle Probleme im Gesundheitswesen, die von der Forschung kaum beeinflusst werden können. So ist hinlänglich bekannt, dass die praktische Realisierung gesundheitsökonomischer Erkenntnisse weitgehend davon abhängt, ob es geeignete Anreizsysteme gibt, welche die Leistungsanbieter zum Einsatz effizienterer Methoden und damit zu einem Abgehen von ihrem bislang gewohnten Vorgehen bewegen [17]. Wie schwierig es ist, solche Anreizsysteme zu finden, zeigt die gegenwärtige Debatte um die Einführung der DRGs. Während die klassischen stationären Pflegesätze einen Anreiz zur Ausweitung der Behandlungsdauer setzen, motivieren die neuen DRGs zwar zu einer Verkürzung der stationären Verweildauer, aber auch zu einer Ausweitung der Behandlungsfälle. Die angestrebten kurzen Krankenhausaufenthalte können sich zudem negativ auf die Behandlungsqualität auswirken, da sie vermehrte Rückfälle und eine erhöhte Mortalität im ambulanten Bereich nach sich ziehen können sowie zu einer Zunahme der Verlegungen in Pflegeeinrichtungen führen [18]. Daher wird von vielen schon jetzt bezweifelt, ob die Einführung der DRGs tatsächlich eine Reduktion der GKVAusgaben ermöglichen wird $[19,20]$.

Ein anderes Hemmnis bei der praktischen Umsetzung gesundheitsökonomischer Erkenntnisse ist die im Gesundheitssektor häufig anzutreffende separate Budgetverantwortung unterschiedlicher Kostenträger [21]. Sie kann beispielsweise dazu führen, dass ein neues Therapiekonzept, das die Arbeitsfähigkeit erwerbsunfähiger Patienten wieder herstellt, bei den Krankenkassen trotz einer insgesamt positiven Kosten-Nutzen-Relation nur geringes Interesse findet, weil die Kassen zwar die Kosten für diese Maßnahme tragen müssten, aber nur die Rentenversicherungsträger davon profitieren würden. Diese paradoxe Situation wird in Deutschland noch dadurch verstärkt, dass es zwischen den rund 350 gesetzlichen Krankenkassen nur einen Preiswettbewerb, aber kaum einen Leistungswettbewerb gibt, da 97\% der Kassenleistungen gesetzlich vorgeschrieben sind und somit wenig Raum für ein eigenes Angebotsprofil bleibt.

Die erwähnten Kritikpunkte zeigen, dass sowohl die strukturellen Rahmenbedingungen im Gesundheitswesen als auch die wissenschaftliche Qualität der Untersuchungen verbessert werden müssen, wenn es gelingen soll, gesundheitsökonomische Erkenntnisse künftig auf einer breiteren Basis in die Praxis umzusetzen.

Trotz der diskutierten Einschränkungen bleibt jedoch unbestritten, dass gesundheitsökonomische Untersuchungen sinnvoll und wichtig sind, weil sie eine Basis für rational begründete Entscheidungen im Gesundheitswesen darstellen und die Kostensowie die Gesundheitsimplikationen von Handlungsalternativen aufzeigen [17,22]. Damit gesundheitsökonomische Erkenntnisse aber tatsächlich zur Optimierung der Ressourcenallokation eingesetzt und nicht zur Sanktionierung staatlich verordneter Sparmaßnahmen missbraucht werden, sollten die Wissenschaftler sich stärker für die Umsetzung ihrer Ergebnisse engagieren und sich nicht darauf berufen, dass sie nur die Daten liefern und alles, was weiter damit geschieht, Sache der Politik ist. Sonst besteht die Gefahr, dass das noch junge Fachgebiet zu einer von der Versorgungsrealität abgekoppelten Theorie wird, die vor lauter Gesundheitsökonomie die Krankheit der Patienten aus den Augen verliert.

\section{Literatur}

${ }^{1}$ Gäfgen G. Ökonomie des Gesundheitswesens. Jahrestagung des Vereins für Socialpolitik in Saarbrücken 1985. Berlin: Duncker \& Humblot, 1986

${ }^{2}$ Schulenburg JM Graf vd . Die Entwicklung der Gesundheitsökonomie und ihre methodischen Ansätze. In: Schöffski O, Glaser P, Graf vd Schulenburg JM: Gesundheitsökonomische Evaluationen: Grundlagen und Standortbestimmung. Berlin: Springer, 1998: 15-23

${ }^{3}$ Leidl R. Gesundheitsökonomie und Public Health. In: Walter U, Paris W: Public Health. Gesundheit im Mittelpunkt. Meran: Alfred \& Söhne, 1996: $78-82$

${ }^{4}$ Lauterbach KW. Gesundheitsökonomie als Teil der Qualitätsverbesserung. In: Lauterbach KW, Schrappe M: Gesundheitsökonomie, Qualitätsmanagement und Evidence-based Medicine. Stuttgart: Schattauer, 2001: $121-123$

${ }^{5}$ Dietrich ES. Grundlagen der Pharmakoepidemiologie und Pharmakoökonomie. Eschborn: Govi, 2002

${ }^{6}$ Melchinger H. Ambulante Soziotherapie - Evaluation und analytische Auswertung des Modellprojekts „Ambulante Rehabilitation psychisch Kranker“ der Spitzenverbände der gesetzlichen Krankenkassen. Bd. 115, Schriftenreihe des Bundesministeriums für Gesundheit. BadenBaden: Nomos, 1999

${ }^{7}$ Nowack N. Ambulante Soziotherapie. DÄ 2002; 99 (45): B2532 - 2533

${ }^{8}$ Gerard K, Mooney G. QALY league tables: handle with care. Health Econ 1992; 2: 59-64 
${ }^{9}$ Mason J, Drummond M, Torrance G. Some guidelines on the use of cost-effectiveness league tables. BMJ 1993; 306: 570-572

${ }^{10}$ Rössler W, Salize J, Knapp M. Die Kosten der Schizophrenie. Fortschr Neurol Psychiat 1998; 66: 496-504

${ }^{11}$ Grof P. Has the effectiveness of lithium changed? Impact of the variety of lithium's effects. Neuropsychopharmacology 1998; 19 (3): 183-188

12 Guscott R, Taylor L. Lithium prophylaxis in recurrent affective illness. Efficacy, effectiveness and efficiency. Br J Psychiatry 1994; 164 (6): $741-746$

${ }^{13}$ Markar HR, Mander AJ. Efficacy of lithium prophylaxis in clinical practice. Br J Psychiatry 1989; 155: 496-500

${ }^{14}$ Eberhart L, Schmude M, Geldner G. Der Arzt unter zunehmendem Kostendruck. DÄ 2002; 99 (36): B1979

${ }^{15}$ Schöffski O. Design pharmakoökonomischer Studien. Gibt es Standardisierungen? Krankenhauspharmazie 1995; 16 (3): 89-94

${ }^{16}$ Drummond M, Brandt A, Luce B, Rovira J. Standardizing methodologies for economic evaluation in health care. Practice, problems, and potential. Int J Technol Assess Health Care 1993; 9 (1): 26-36

17 Leidl R. Der Effizienz auf der Spur: Eine Einführung in die ökonomische Evaluation. In: Schwartz FW: Das Public Health Buch: Gesund- heit und Gesundheitswesen. München: Urban \& Schwarzenberg, 1998: 346 - 369

${ }^{18}$ Metersky ML, Tate JP, Fine MJ, Petrillo MK, Meehan TP. Temporal trends in outcomes of older patients with pneumonia. Arch Intern Med 2000; 160: 3385-3391

${ }^{19}$ Frantz E, Fleck E. Neues Entgeltsystem - ein großer Wurf? DÄ 2001; $98(1-2)$ : B19-B21

${ }^{20}$ Clade H. Krankenhausfinanzierung. Falscher Ansatz mit Kostenwirkungen. DÄ 2002; 99 (38): B2092

${ }^{21}$ Sloan FA, Conover CJ. The use of cost-effectiveness/cost-benefit analysis in actual decision making: current status and prospects. In: Sloan FA: Valuing health care. Costs, benefits, and effectiveness of pharmaceuticals and other medical technologies. Cambridge: Cambridge University Press, 1995: 207-232

${ }^{22}$ Schöffski O, Greiner W. Das QALY-Konzept zur Verknüpfung von Lebensqualitätseffekten mit ökonomischen Daten. In: Schöffski O, Glaser P, Graf vd Schulenburg JM: Gesundheitsökonomische Evaluationen: Grundlagen und Standortbestimmung. Berlin: Springer, 1998: $203-222$ 